\title{
REIDEMEISTER CLASSES IN SOME WEAKLY BRANCH GROUPS
}

\author{
EVGENIJ TROITSKY
}

\begin{abstract}
We prove that a saturated weakly branch group $G$ has the property $R_{\infty}$ (any automorphism $\phi: G \rightarrow G$ has infinite Reidemeister number) in each of the following cases:

1) any element of $\operatorname{Out}(G)$ has finite order;

2) for any $\phi$ the number of orbits on levels of the tree automorphism $t$ inducing $\phi$ is uniformly bounded and $G$ is weakly stabilizer transitive;

3) $G$ is finitely generated, prime-branching, and weakly stabilizer transitive with some non-abelian stabilizers (with no restrictions on automorphisms).

Some related facts and generalizations are proved.
\end{abstract}

\section{INTRODUCTION}

Consider an automorphism $\phi: G \rightarrow G$ of a (countable discrete) group. The Reidemeister number $R(\phi)$ is the number of its Reidemeister or twisted conjugacy classes, i.e. the classes of the following equivalence relation: $g \sim h g \phi\left(h^{-1}\right), h, g \in G$. The Reidemeister class of an element $g$ we denote by $\{g\}_{\phi}$.

A group has the $R_{\infty}$ property if $R(\phi)=\infty$ for any automorphism $\phi: G \rightarrow G$. The problem of determining of groups having the $R_{\infty}$ property was raised by A.Fel'shtyn and co-authors in relation with an older conjecture by A.Fel'shtyn and R.Hill [5]: $R(\phi)$ is equal to the number of fixed points of the associated homeomorphism $\widehat{\phi}$ of the unitary dual $\widehat{G}$, if one of these numbers is finite. This conjecture is called TBFT (twisted Burnside-Frobenius theorem), because it generalizes to infinite groups and to the twisted case the classical Burnside-Frobenius theorem: the number of conjugacy classes of a finite group is equal to the number of equivalence classes of its irreducible representations. The question about TBFT formally has a positive answer for $R_{\infty}$ groups. So, the $R_{\infty}$ problem is in some sense complementary to the TBFT.

The TBFT conjecture was proved for finite, abelian and abelian-by-finite groups [5]. The further development, examples, counterexamples and modifications can be found in [11, 9, $10,25,26,27]$.

The property $R_{\infty}$ was proved and disproved for many groups and the number of papers on the subject and related questions is too large to list all of them and we restrict ourselves to giving reference to several papers and bibliography overview therein: $[24,1,19,20,12,13$, 21, 17, 2, 8]. Dynamical aspects of Reidemeister numbers are discussed in [4]. Some direct topological consequences of the property $R_{\infty}$ for Jiang-type spaces are discussed in [13].

In [6] the $R_{\infty}$ property was proved for a wide class of saturated weakly branch groups.

In the present paper we develop these results and prove in Theorem 2.2 that if any automorphism of a saturated weakly branch group $G$ is a composition of an inner automorphism

2000 Mathematics Subject Classification. 20E45; 20B35; 20F28; 20F65 .

Key words and phrases. Reidemeister number, $R_{\infty}$-group, twisted conjugacy class, residually finite group, weakly branch group.

This work is supported by the Russian Science Foundation under grant 16-11-10018. 
and of a finite order automorphism, then $G$ has the property $R_{\infty}$. In particular, this theorem holds for the Grigorchuk group and for the Gupta-Sidki group. In some specific cases the result can be obtained from [17].

We introduce the property WST (Definition 1.7) and prove that if for any automorphism $\phi$ of a saturated weakly branch WST group $G$, induced by an automorphism $t$ of the tree, i.e. $\phi(g)=t g t^{-1}$, restrictions of $t$ on levels have a uniformly bounded number of orbits, then $G$ has the property $R_{\infty}$ (Theorem 3.3).

In Theorem 4.2 we prove the $R_{\infty}$ property without any restrictions on the structure of the automorphism group of a finitely generated saturated weakly branch WST group $G$, but with the restriction on branching numbers to be prime and with an additional restriction on stabilizers.

We prove that a saturated weakly branch group on a spherically symmetric tree, such that any level stabilizer contains an odd permutation at some level, is an $R_{\infty}$ group (Theorem $5.2)$.

Acknowledgement: The author is indebted to A. Fel'shtyn and the MPIM for helpful discussions in the Max-Planck Institute for Mathematics (Bonn) in February, 2017, to V. Manuilov for useful suggestions, and to A. Jaikin-Zapirain for a bibliography reference.

This work is supported by the Russian Science Foundation under grant 16-11-10018.

\section{Preliminaries}

First, we recall some necessary facts about Reidemeister classes.

Lemma 1.1. Any Reidemeister class of $\phi$ is formed by some $\phi$-orbits.

Proof. Indeed, $\phi(g)=g^{-1} g \phi(g)$.

Definition 1.2. Denote by $\tau_{g}$ the inner automorphism: $\tau_{g}(x)=g x g^{-1}$.

From the equality

$$
x y \varphi\left(x^{-1}\right) g=x(y g) g^{-1} \varphi\left(x^{-1}\right) g=x(y g)\left(\tau_{g^{-1}} \circ \varphi\right)\left(x^{-1}\right)
$$

we immediately obtain the following statement.

Lemma 1.3. A right shift by $g \in G$ maps Reidemeister classes of $\phi$ onto Reidemeister classes of $\tau_{g^{-1}} \circ \varphi$, In particular, $R\left(\tau_{g} \circ \phi\right)=R(\phi)$.

Lemma 1.4 ([7, Prop. 3.4]). Suppose, $\phi$ is an automorphism of a finitely generated residually finite group. Let $R(\phi)=r<\infty$. Then the number of fixed elements of $\phi$ is bounded by $a$ function depending only on $r$.

Now we pass to groups acting on trees and give some known and new definitions and facts.

Let $\mathcal{T}$ be a spherically symmetric rooted tree. This means that all vertexes of the same level have the same number of immediate descendants (branching index).

Denote by $D(v)$ the set of immediate descendants of a vertex $v \in \mathcal{T}$.

A group $G$ acting faithfully on a rooted tree is a weakly branch group, if for any vertex $v$ of $\mathcal{T}$, there exists an element of $G$ which acts nontrivially on the subtree $\mathcal{T}_{v}$ with the root vertex $v$ and trivially outside this subtree. In other words, the rigid stabilizer Rist $v$ of any vertex $v$ is non-trivial.

Evidently a faithful tree group is residually finite. 
We will denote by $\operatorname{St}(v)$ the stabilizer of a vertex $v \in \mathcal{T}$; and by $\mathrm{St}_{j}$ the stabilizer of level $L_{j}$, i.e. $\mathrm{St}_{j}=\cap_{v \in L_{j}} \operatorname{St}(v)$.

A group $G$ is saturated if, for every positive integer $n$, there exists a characteristic subgroup $H_{n} \subset G$ acting trivially on the $n$-th level of $\mathcal{T}$ and level transitive on any subtree $\mathcal{T}_{v}$ with $v$ in the $n$-th level.

Theorem 1.5 ([18]). Suppose, $G$ is a saturated weakly branch group on a tree $\mathcal{T}$. Then its automorphism group Aut $G$ coincides with the normalizer of $G$ in the full group of isometries $I s o(\mathcal{T})$ of the rooted tree $\mathcal{T}$ : every automorphism $\phi$ of the group $G$ is induced by the conjugation by an element $t$ from the normalizer and the centralizer of $G$ in $I \operatorname{so}(\mathcal{T})$ is trivial.

Definition 1.6. For a group $G$ acting on $\mathcal{T}$ and any vertex $v \in \mathcal{T}$ denote by $G_{\{v\}}$ the subgroup of all elements $g \in G$ fixing $v$ and all vertexes of $\mathcal{T}$ from the next level, except of immediate descendants of $v$.

In other words, if $v \in L_{j}$, then

$$
G_{\{v\}}=\bigcap_{w \in L_{j}, w \neq v} \bigcap_{u \in D(w)} \operatorname{St}(u)
$$

Thus,

$$
\operatorname{Rist}_{v} \subset G_{\{v\}} \subset \mathrm{St}_{j} .
$$

Definition 1.7. We call a group $G$ acting on $\mathcal{T}$ weakly stabilizer transitive (WST) if for any vertex $v$ one can find a vertex $v_{0} \in \mathcal{T}_{v}$ such that $G_{\left\{v_{0}\right\}}$ acts transitively on immediate descendants of $v_{0}$.

Remark 1.8. If $G$ acts level-transitively, then $G_{\{v\}}$ are pairwise isomorphic for $v$ from the same level. Also, they pairwise commute and we can introduce the following well-defined group $\Gamma_{\{i\}}$.

Definition 1.9. Denote

$$
\Gamma_{\{i\}}:=\prod_{v \in L_{i-1}} p_{i}\left(G_{\{v\}}\right),
$$

where $p_{i}: G \rightarrow G / \mathrm{St}_{i}$ is the natural projection.

Let $t$ be an automorphism of a tree $\mathcal{T}$. Let $\operatorname{Orb}_{i}(t)$ be the number of orbits of $t$ at the level $L_{i}$. Evidently,

1) $\operatorname{Orb}_{i}(t)$ is a not-decreasing function of $i$;

2) a fixed vertex of $t$ may be only a successor of a fixed vertex;

$3)$ if there is a fixed vertex at the level $i+1$, then $\operatorname{Orb}_{i+1}(t)>\operatorname{Orb}_{i}(t)$.

So, we have two possibilities:

(a) $\operatorname{Orb}_{i}(t) \longrightarrow \infty$ as $i \rightarrow \infty$;

(b) $\operatorname{Orb}_{i}(t)$ is bounded. In this case, there is no fixed vertices starting some level, by 3 ) above.

Finally, we will need the following statement from the Galois theory (see, e.g. [3, Sect. $3.5])$ :

Lemma 1.10. A solvable transitive subgroup of the symmetric group $S_{p}$, where $p$ is prime, is isomorphic either to $\mathbb{Z}_{p}$, or to $\mathbb{Z}_{p} \rtimes \mathbb{Z}_{p-1}$. In particular, it is either abelian, or contains an odd permutation (a generator of $\mathbb{Z}_{p-1}$ ). 


\section{Finite order AUtomorphisms AND AROUnd}

Lemma 2.1. Let $\phi: G \rightarrow G$ be an automorphism of oder $n<\infty$ of a weakly branch group with $\phi(g)=t_{g} t^{-1}$. Then there exists $j_{0}$ such that for any $j \geqslant j_{0}$ there exists an element $g_{j} \in \mathrm{St}_{j}$ and a number $i>j$ such that $\left\{g_{j}\right\}_{\phi} \cap \mathrm{St}_{i}=\varnothing$.

Proof. It is sufficient to find an element $g_{j}$ such that $h g_{j} t h^{-1} t^{-1} \neq e$ at the level $L_{i}$ for any $h \in G$, or equivalently

$$
g_{j} t \neq h^{-1} t h .
$$

By the condition, $t$ has at some level an orbit of length $n$, and does not have a longer orbit. Take for $j_{0}$ the first time when $t$ has on $L_{j_{0}}$ an orbit of length $n$. Then the orbits of successors also will have the length $n$. Consider any $j \geqslant j_{0}$ and an orbit of length $n$ in $L_{j}$. Let $v_{0} \in L_{j}$ be a vertex from this orbit. Using the weak branching property we can find a non-trivial element $g_{j} \in \operatorname{Rist}\left(v_{0}\right)$. Let $i$ be the first level where $g_{j}$ acts non trivially, say at $v \in \mathcal{T}_{v_{0}}$ (see Fig. 1). Then

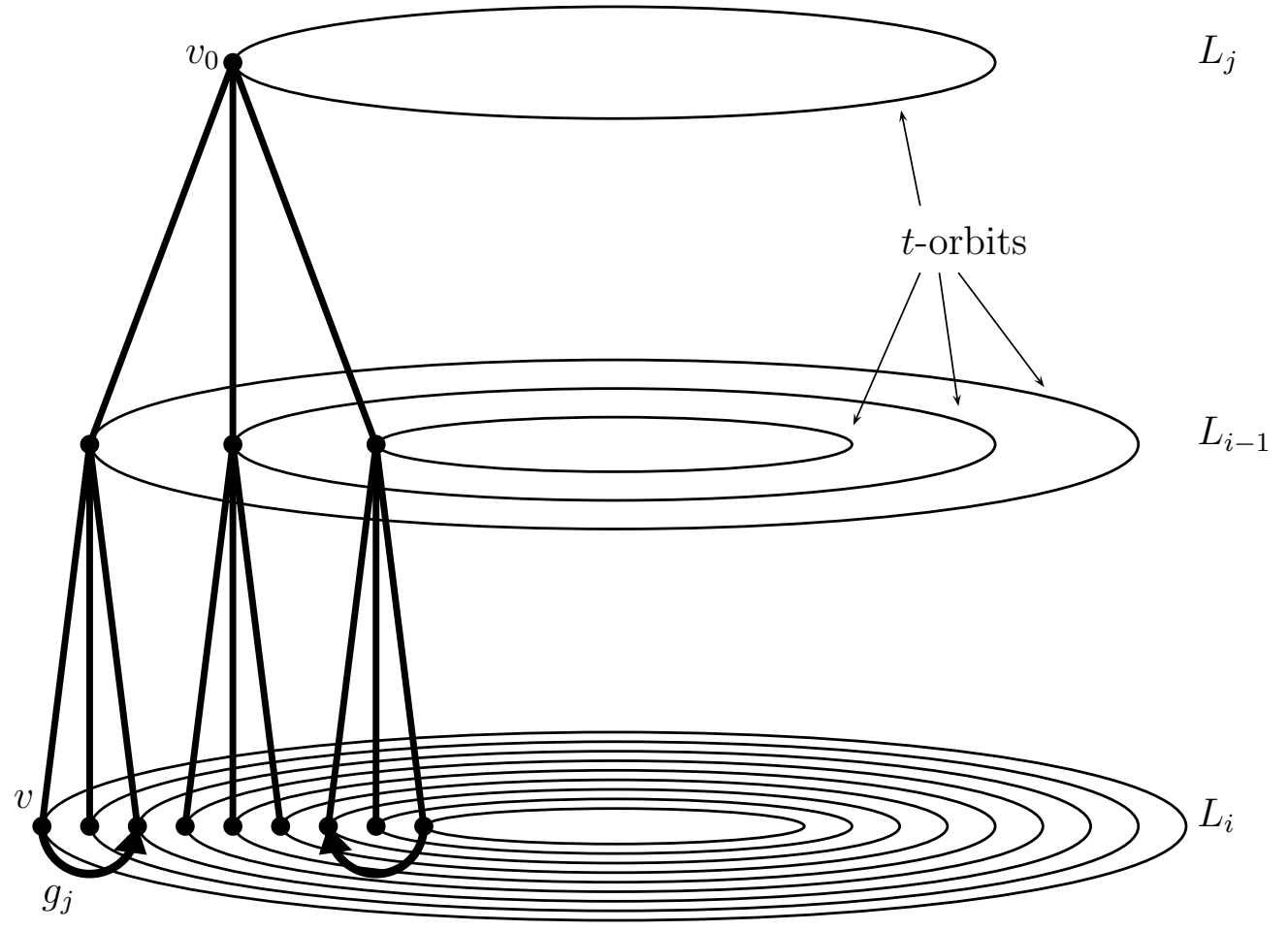

FIGURE 1 .

$$
\left(g_{j} t\right)^{n}(v)=g_{j} t^{n}(v)=g_{j}(v) \neq v,
$$

because the $t$-orbit of $v$ has the form $v, t(v), \ldots, t^{n-1}(v), t^{n}(v)=v$ and $t(v), \ldots, t^{n-1}(v) \notin \mathcal{T}_{v_{0}}$ implying $g t(v)=t(v), \ldots, g t^{n-1}(v)=t^{n-1}(v)$. So, $g_{j} t$ has an orbit of length $>n$ and can not be conjugate to $t$ at the level $L_{i}$. We obtain (1).

Theorem 2.2. Suppose, $G$ is a saturated weakly branch group and each automorphism from $\operatorname{Out}(G)$ is of finite order. Then $G$ has the $R_{\infty}$ property. 
Proof. By Lemma 1.3 it is sufficient to verify $R(\phi)=\infty$ for some $\phi$ of finite order $n$.

By Theorem $1.5 \phi(g)=t g t^{-1}$ for an automorphism $t$ of the tree. Then Lemma 2.1 gives inductively an infinite sequence of representatives of distinct Reidemeister classes. Thus $R(\phi)=\infty$.

Example 2.3. The most studied branch groups - the Grigorchuk group [14] and the GuptaSidki group [16] - have outer automorphisms of finite order [15, 23].

Example 2.4. A more evident example is the group of all isometries of a symmetric rooted tree. In this case all automorphisms are inner.

\section{Finite NUMBER OF ORBITS}

Now we consider the opposite case, when the number of orbits $t$ on $L_{i}$ is uniformly bounded. We will need to restrict ourselves to the WST case.

Lemma 3.1. Let $\phi: G \rightarrow G$ be an automorphism of a WST group with $\phi(g)=t_{g} t^{-1}$, where $t$ is an automorphism of the tree $\mathcal{T}$. Suppose, $t$ satisfies (b) above, namely, $\max _{i} \operatorname{Orb}_{i}(t)=$ $M<\infty$. Then there exists $j_{0}$ such that for any $j \geqslant j_{0}$ there exists an element $g_{j} \in \mathrm{St}_{j}$ and a number $i>j$ such that $\left\{g_{j}\right\}_{\phi} \cap \mathrm{St}_{i}=\varnothing$.

Proof. Let $j_{0}$ be the level of stabilization of the number of orbits, i.e., $\operatorname{Orb}_{j_{0}-1}(t)<M$ and $\operatorname{Orb}_{j_{0}}(t)=M$, hence $\operatorname{Orb}_{j}(t)=M$ for any $j \geqslant j_{0}$. Note that the lengths of orbits of $t$ at next levels, are the multiples of lengths of orbits on $L_{j_{0}}$ (with the coefficient equal to the appropriate product of branching numbers) and an orbit of smallest length (not unique generally) lies under a smallest orbit on $L_{j_{0}}$.

Now take an arbitrary $j \geqslant j_{0}$ and consider an orbit of $t$ of the smallest size on $L_{j}$. Let $v$ be a vertex from this orbit, and find by the WST property an element $v_{0} \in \mathcal{T}_{v}, v_{0} \in L_{i-1}$ for some $i$, with a transitive action of $G_{\left\{v_{0}\right\}}$ on its immediate successors. Let $v_{1}$ be one of these successors. Then, as it was explained, its $t$-orbit has the smallest length among the orbits on $L_{i}$. This length is equal to $m \cdot b$, where $m$ is the length of $t$-orbit of $v_{0}$ and $b$ is the branching number of $v_{0}$. Choose $g_{j} \in G_{\left\{v_{0}\right\}}$ such that $g_{j} t^{m}\left(v_{1}\right)=v_{1}$ (see Fig. 2). By the definition of $G_{\left\{v_{0}\right\}}$,

$$
g_{j} t\left(v_{1}\right)=t\left(v_{1}\right), \quad\left(g_{j} t\right)^{2}\left(v_{1}\right)=t^{2}\left(v_{1}\right), \ldots \quad\left(g_{j} t\right)^{m}\left(v_{1}\right)=g_{j} t^{m}\left(v_{1}\right)=v_{1} .
$$

Hence, the smallest length of a $\left(g_{j} t\right)$-orbit on $L_{i}$ is $m<m \cdot b=$ the smallest length of a $t$-orbit on $L_{i}$. Thus, $g_{i} t$ and $t$ can not be conjugate and we arrive to (1) and the same argument as in the beginning of the proof of Lemma 2.1, completes the proof.

Remark 3.2. By Lemma $1.1 R(\phi)<\infty$, if the number of $\phi$-orbits is finite. But the number of $\phi$-orbits in $G$ is rather weakly related to the number of $t$-orbits on $\mathcal{T}$. For example, for $t=\mathrm{Id}$, this depends on "how saturated $G$ is".

Similarly to the proof of Theorem 2.2, one can deduce from Lemma 3.1 the following statement.

Theorem 3.3. If a saturated weakly branch group $G$ is a WST group and each its non-trivial outer automorphism has the properties from Lemma 3.1, then $G$ is an $R_{\infty}$ group.

Example 3.4. We do not expect interesting examples of groups here, moreover, we need the results of this section mostly as a tool for proofs with using for some automorphisms in the next section (case b) below).

Nevertheless, Example 2.4 works here too. 


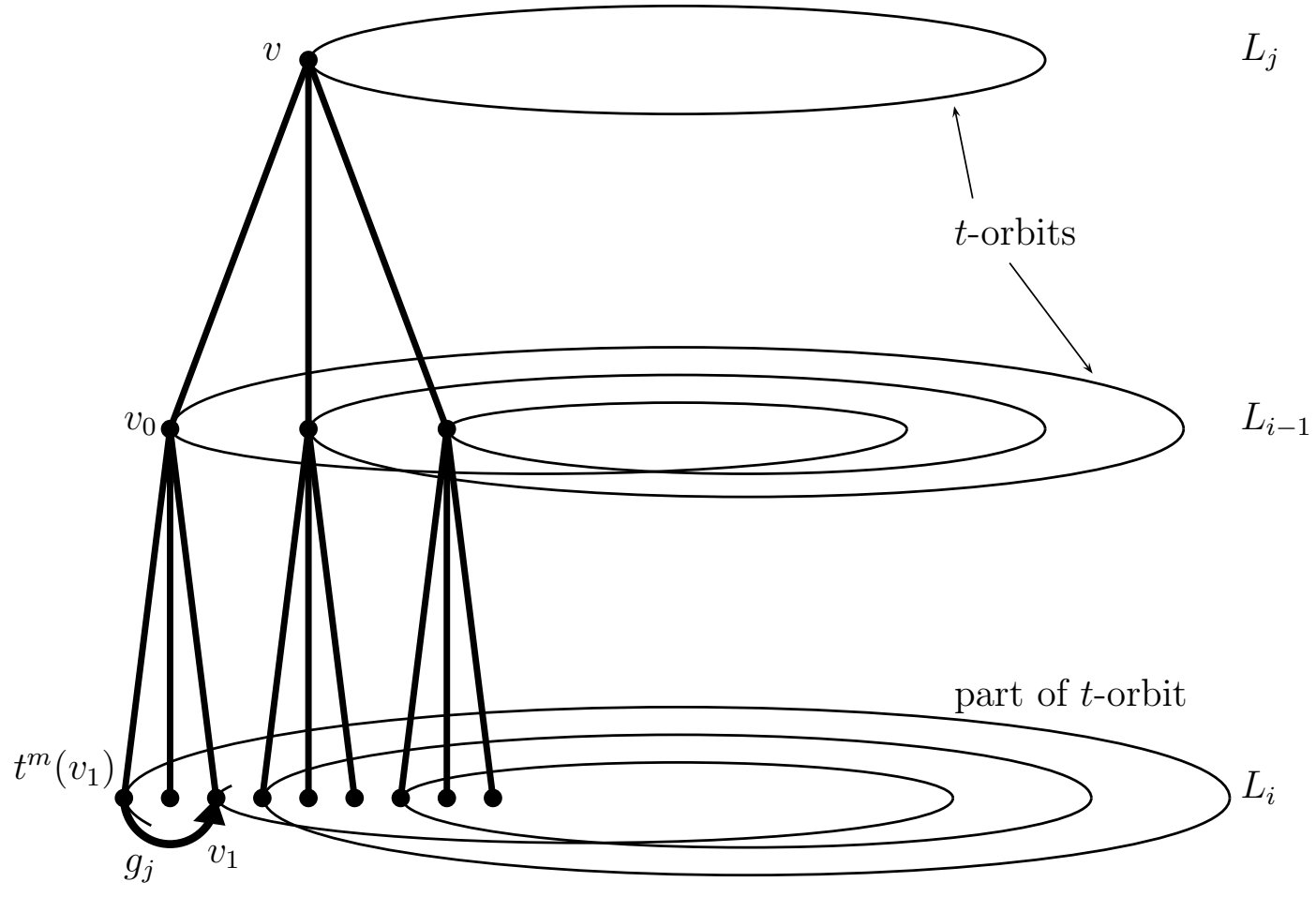

FiguRE 2.

4. The GENERAL CASE

Lemma 4.1. Let $\phi: G \rightarrow G$ be an automorphism of a group $G$ acting level-transitively on a spherically symmetric tree $\mathcal{T}$, with $\phi(g)=\operatorname{tgt}^{-1}$. Suppose,

(1) $G$ is finitely generated;

(2) $G$ is a WST group;

(3) moreover, for an infinite subsequence $\left\{i_{k}\right\}$ of the sequence of levels, arising as transitivity levels in the definition of WST, the corresponding group $\Gamma_{\{i\}}$ (see Def. 1.9) is not abelian;

(4) branching numbers are prime (may be distinct for distinct levels).

Suppose, $R(\phi)<\infty$. Then there exists $j_{0}$ such that for any $j \geqslant j_{0}$ there exists an element $g_{j} \in \mathrm{St}_{j}$ and a number $i>j$ such that $\left\{g_{j}\right\}_{\phi} \cap \mathrm{St}_{i}=\varnothing$.

Proof. As in the proof of Lemma 2.1, it is sufficient to find an element $g_{j} \in \mathrm{St}_{j}$ such that at the level $L_{i}$ for any $h \in G$

$$
g_{j} \neq h^{-1} t h t^{-1}
$$

Consider two cases:

a) $\operatorname{Orb}_{i}(t) \rightarrow \infty$;

b) $\operatorname{Orb}_{i}(t)$ is bounded.

Case a). Since $R(\phi)<\infty$ and $G$ is finitely generated, by Lemma 1.4 the number of $\phi$-fixed elements for the quotient $G / S t_{i}$ is strictly less $\operatorname{Orb}_{i-1}(t)$ at each level $i$ greater some $j_{0}$. 
Now for any $j>j_{0}$, let $i-1>j$ be the number of a level with transitive action of $G_{\{v\}}$ for any $v \in L_{i-1}$ (see Remark 1.8) such that $\Gamma_{\{i\}}$ is not abelian.

Each of the above-mentioned $\phi$-fixed elements (except of $e$ ) acts non-trivially at some vertex $w_{s}$. Thus an element, which fixes these vertexes, is not $\phi$-fixed. Hence there exists $v_{0} \in L_{i-1}$ such that for any $v$ in its $t$-orbit, $p_{i}\left(G_{\{v\}}\right)$ does not contain $\phi$-fixed points, where $p_{i}: G \rightarrow G / S t_{i}$. Suppose, the $t$-orbit of $v_{0}$ has some length $k$ ( $k=1$ can occur in particular). Then

$$
p_{i} G_{\left\{t^{m}\left(v_{0}\right)\right\}}=t^{m} p_{i} G_{\left\{v_{0}\right\}} t^{-m}, \quad m=0,1, \ldots, k-1 .
$$

Evidently, elements of these groups commute, and we can form a group

$$
\Gamma:=p_{i}\left(G_{\left\{v_{0}\right\}}\right) \cdots p_{i} G_{\left\{t^{k-1}\left(v_{0}\right)\right\}}
$$

with an action of $\phi$. Each $\gamma \in \Gamma$ acts trivially on all $w_{s}$. Hence, $\Gamma$ has no nontrivial $\phi$-fixed elements. So, $\Gamma$ is a subgroup with a fixed-point-free automorphism $\phi$. Then it is solvable by $[22]$.

Hence, its subgroup $p_{i}\left(G_{\left\{v_{0}\right\}}\right)$ is also solvable. It is a transitive subgroup of the symmetric group $S_{p}$, where $p$ is the prime branching number for vertexes from $L_{i-1}$. Then, by Lemma 1.10 , it is either abelian, or contains an odd permutation $p_{i}\left(g_{j}\right) \notin A_{p}, g_{j} \in G_{\left\{v_{0}\right\}}$. In the first case, $\Gamma_{\{i\}}$ is abelian in a contradiction with the supposition. In the second case, $g_{j}$ is trivial on $L_{i}$ except the successors of $v_{0}$. So it is an odd permutation on the entire $L_{i}$, while $h^{-1} t h t^{-1}$ is an even one. This gives (3).

Case b). This case immediately follows from Lemma 3.1.

Similarly to the proof of Theorem 2.2 we obtain from Lemma 4.1 the following statement.

Theorem 4.2. Suppose, $G$ is a finitely generated saturated weakly branch WST group on a spherically symmetric tree with prime branching numbers and an infinite sequence of nonabelian $\Gamma_{\{i\}}$ (i.e. satisfying the suppositions of Lemma 4.1). Then $G$ is an $R_{\infty}$ group.

Remark 4.3. Reasonable examples will be given in the next section for a version of this statement, namely Theorem 5.2.

\section{Some Generalizations}

Evidently the above statements can be easily extended to some more general cases (with more complicated formulations).

For example, Theorem 2.2 can be evidently generalized in the following way.

Theorem 5.1. Suppose, $G$ is a weakly branch group and each automorphism from $\operatorname{Out}(G)$ is of finite order and defined by an automorphism of the tree. Then $G$ has the $R_{\infty}$ property.

Now we will give another version of Theorem 4.2.

Theorem 5.2. Suppose, $G$ is a saturated weakly branch group on a spherically symmetric tree, such that for any $j, \mathrm{St}_{j}$ contains an element $g_{j}$ defining an odd permutation at some level $j_{0}>j$. Then $G$ is an $R_{\infty}$ group.

Proof. Indeed, (3) keeps, because $h^{-1} t h t^{-1}$ is an even permutation and $g_{j}$ is an odd permutation at the level $j_{0}$.

Example 5.3. The full isometry group as in Example 2.4 satisfies the conditions of Theorem 5.2 . 
Example 5.4. Consider a saturated weakly branch group $G$ and consider a group $\Gamma$ generated by $G$ and an infinite series of isometries $g_{j}$, e.g., transpositions of two neighbouring elements at level $L_{j+1}$ and somehow defined at their successors. Then $\Gamma$ satisfies the conditions of Theorem 5.2 .

\section{REFERENCES}

[1] Collin Bleak, Alexander Fel'shtyn, and Daciberg L. Gonçalves. Twisted conjugacy classes in R. Thompson's group F. Pacific J. Math. 238, No. 1, 1-6, 2008.

[2] Karel Dekimpe and Daciberg Gonçalves. The $R_{\infty}$ property for free groups, free nilpotent groups and free solvable groups. Bull. Lond. Math. Soc. 46, No. 4, 737-746, 2014.

[3] John D. Dixon and Brian Mortimer. Permutation groups, volume 163 of Graduate Texts in Mathematics. Springer-Verlag, New York, 1996.

[4] Alexander Fel'shtyn. Dynamical zeta functions, Nielsen theory and Reidemeister torsion. Mem. Amer. Math. Soc. 147, No. 699, xii+146, 2000.

[5] Alexander Fel'shtyn and Richard Hill. The Reidemeister zeta function with applications to Nielsen theory and a connection with Reidemeister torsion. K-Theory 8, No. 4, 367-393, 1994.

[6] Alexander Fel'shtyn, Yuri Leonov, and Evgenij Troitsky. Twisted conjugacy classes in saturated weakly branch groups. Geometriae Dedicata 134, 61-73, 2008.

[7] Alexander Fel'shtyn, Nikita Luchnikov, and Evgenij Troitsky. Twisted inner representations. Russian Journal of Mathematical Physics 22, No. 3, 301-306, 2015.

[8] Alexander Fel'shtyn and Timur Nasybullov. The $R_{\infty}$ and $S_{\infty}$ properties for linear algebraic groups. J. Group Theory 19, No. 5, 901-921, 2016.

[9] Alexander Fel'shtyn and Evgenij Troitsky. Twisted Burnside-Frobenius theory for discrete groups. J. Reine Angew. Math. 613, 193-210, 2007.

[10] Alexander Fel'shtyn and Evgenij Troitsky. Aspects of the property $R_{\infty}$. J. Group Theory 18, No. 6, 1021-1034, 2015.

[11] Alexander Fel'shtyn, Evgenij Troitsky, and Anatoly Vershik. Twisted Burnside theorem for type $\mathrm{II}_{1}$ groups: an example. Math. Res. Lett. 13, No. 5, 719-728, 2006.

[12] Daciberg Gonçalves and Peter Wong. Twisted conjugacy classes in wreath products. Internat. J. Algebra Comput. 16, No. 5, 875-886, 2006.

[13] Daciberg Gonçalves and Peter Wong. Twisted conjugacy classes in nilpotent groups. J. Reine Angew. Math. 633, 11-27, 2009.

[14] Rostislav I. Grigorchuk. On Burnside's problem on periodic groups. Funct. Anal. Appl. 14, 41-43, 1980.

[15] Rostislav I. Grigorchuk and Said N. Sidki. The group of automorphisms of a 3-generated 2group of intermediate growth. Internat. J. Algebra Comput. 14, No. 5-6, 667-676, 2004. International Conference on Semigroups and Groups in honor of the 65th birthday of Prof. John Rhodes.

[16] Narain Gupta and Said Sidki. On the Burnside problem for periodic groups. Math. Z. 182, 385-388, 1983.

[17] Enrico Jabara. Automorphisms with finite Reidemeister number in residually finite groups. J. Algebra 320, No. 10, 3671-3679, 2008.

[18] Yaroslav LaVreniuk and Volodymyr NeKrashevych. Rigidity of branch groups acting on rooted trees. Geom. Dedicata 89, 159-179, 2002.

[19] T. Mubeena and P. Sankaran. Twisted Conjugacy Classes in Abelian Extensions of Certain Linear Groups. Canad. Math. Bull. 57, No. 1, 132-140, 2014.

[20] Timur R. Nasybullov. Twisted conjugacy classes in general and special linear groups. Algebra Logika 51, No. 3, 331-346, 415, 418, 2012.

[21] Vitaly Roman'kov. Twisted conjugacy classes in nilpotent groups. J. Pure Appl. Algebra 215, No. 4, 664-671, 2011.

[22] Peter Rowley. Finite groups admitting a fixed-point-free automorphism group. J. Algebra 174, No. 2, 724-727, 1995.

[23] SAID SidKI. On a 2-generated infinite 3-group: subgroups and automorphisms. J. Algebra 110, No. 1, $24-55,1987$. 
[24] Jennifer Taback and Peter Wong. Twisted conjugacy and quasi-isometry invariance for generalized solvable Baumslag-Solitar groups. J. Lond. Math. Soc. (2) 75, No. 3, 705-717, 2007.

[25] Evgenij Troitsky. Noncommutative Riesz theorem and weak Burnside type theorem on twisted conjugacy. Funct. Anal. Pril. 40, No. 2, 44-54, 2006. In Russian, English translation: Funct. Anal. Appl. 40 (2006), No. 2, 117-125.

[26] EvgeniJ Troitsky. Two examples related to the twisted Burnside-Frobenius theory for infinitely generated groups. Fundam. Appl. Math. 21, No. 5, 231-239, 2016. (in Russian, English translation in Journal of Mathematical Sciences (New York) to appear, preprint version http://www.mpim-bonn.mpg.de/preblob/5689).

[27] EvgeniJ Troitsky. Reidemeister classes in lamplighter type groups. e-print, 2017. arXiv:1711.09371.

Dept. of Mech. and Math., Moscow State University, 119991 GSP-1 Moscow, Russia

E-mail address: troitsky@mech.math.msu.su

$U R L:$ http://mech.math.msu.su/ troitsky 